\title{
Heartbeat: Telemedicine for echocardiography screening
}

Echocardiography allows accurate, rapid, non-invasive diagnosis of heart disease but is not available in many underserved populations. Nascimento and colleagues ${ }^{1}$ in Brazil tested the hypothesis that telemedicine interpretation of a focused handheld echo study performed by a healthcare provider with condensed imaging training could improve delivery of cardiovascular care to patients in low-resource areas. Remarkably, echo screening of 299 asymptomatic primary care patients identified significant heart disease in 35\%, with a higher prevalence of disease in older patients (figure 1). Comparing screened patients to the 705 patients specifically referred for echocardiography based on clinical symptoms or signs, moderate-severe left ventricular systolic dysfunction was observed in $4.1 \%$ vs $8.1 \%(\mathrm{P}=0.03)$, mitral regurgitation in $8.9 \%$ vs $20.3 \%$ $(\mathrm{P}<0.001)$ and aortic stenosis in $5.4 \%$ vs $4.3 \%(\mathrm{P}=0.51)$. Prevalence of echo-diagnosed heart disease was highest in those over age 65 years.

Peters and Patil ${ }^{2}$ comment that "Cardiac point-of-care ultrasound (POCUS) has been rapidly adopted across a variety of practice settings. The popularity stems from the ability to rapidly assimilate findings at the bedside into a diagnosis and treatment plan." The study by Nascimento and colleagues ${ }^{1}$ is remarkable for the number of patients included, having non-cardiologists perform image acquisition, developing a concentrated imaging training programme and using telemedicine for expert image review. They conclude: "Through high-quality cardiac POCUS, the healthcare community worldwide has a promising new resource to diagnose heart disease."

Discharge against medical advice (AMA) occurs for several reasons, such as lack of trust, poor communication, financial worries, and patient dissatisfaction. Discharge AMA is a particular concern in patients with acute myocardial infarction (AMI) given the known benefits of medical therapy post-discharge. In order to estimate the magnitude of this problem, Kwok and colleagues ${ }^{3}$ looked at data on over 2.6 million patients AMI in the USA

Correspondence to Professor Catherine M Otto Division of Cardiology, University of Washington, Seattle,WA 98195, USA; cmotto@uw.edu

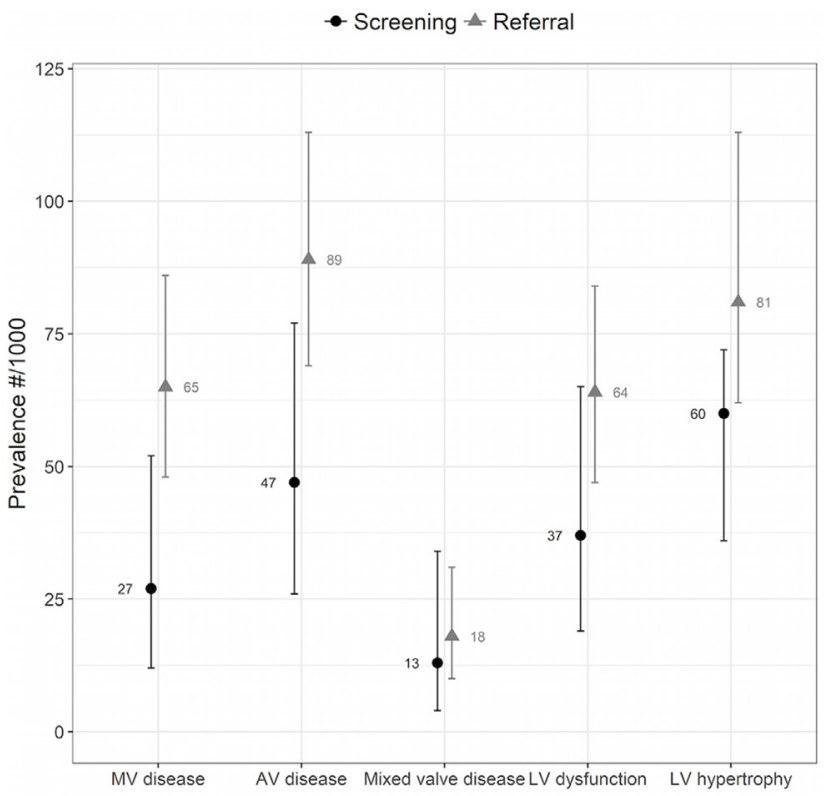

Figure 1 Echocardiographic prevalence of major heart disease requiring priority referral based on the final conclusion of the expert readers, according to study group. AV, aortic valve; $M V$, mitral valve; LV, left ventricle.

National Readmission Database from 2010 to 2014. Overall, the rate of discharge AMA was only $1.5 \%$. However, discharge AMA was associated with a higher rate of 30 day hospital readmissions both for all causes (OR 2.27 95\% CI 2.14 to 2.40 ) and for AMI. Crude readmission rates for AMI were $30.4 \%$ vs $13.4 \%$ with a greater than 3 -fold increased risk in those discharged AMA, even after adjustment for other variables, (OR $3.6595 \%$ CI 3.31 to 4.03 , $\mathrm{P}<0.001$ ) (figure 2).

Alfandre ${ }^{4}$ points out that the study by Kwok and colleagues ${ }^{3}$ identifies several hospitalization-related factors in patients discharged AMA, including the intensity of care with fewer AMA discharges in those who underwent major procedures such as coronary artery bypass grafting surgery, implantation of an automated

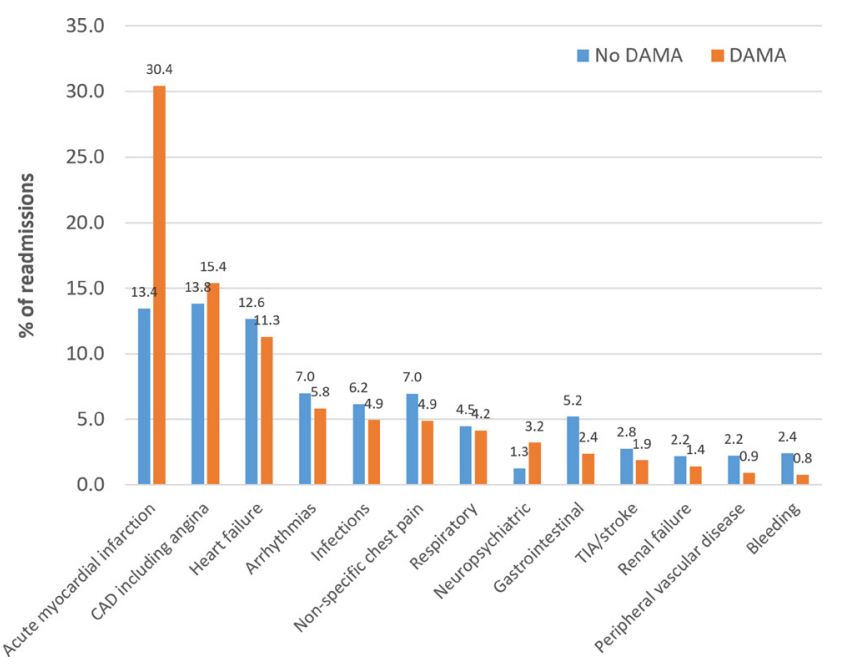

Figure 2 Causes of 30-day unplanned readmissions. DAMA, discharge against medical advice. 


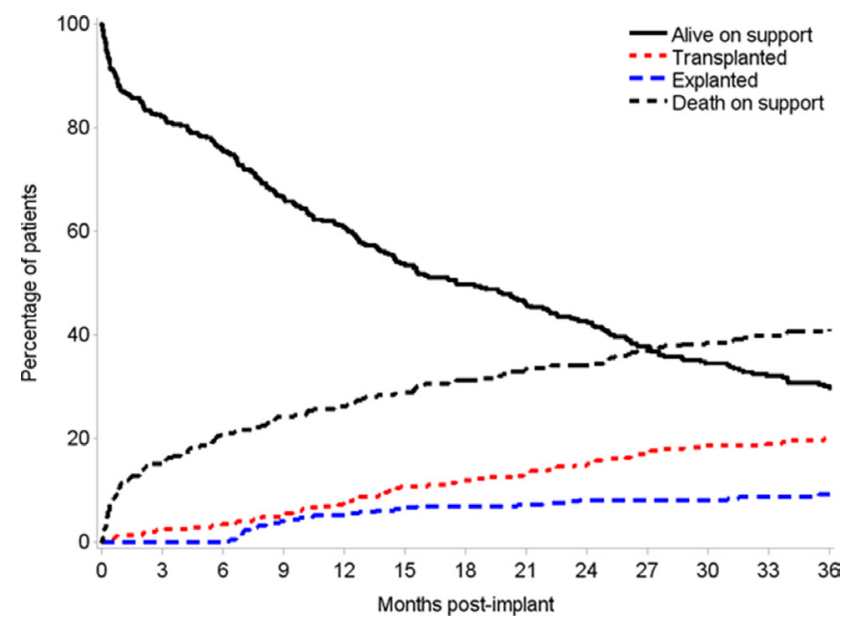

Figure 3 Cumulative incidence of death on left ventricular assist device support, heart transplantation and explant without transplant.

cardiac defibrillation or percutaneous coronary intervention. Discharge AMA also occurred more often at large, urban, non-teaching hospitals. In addition to suggesting avenues of future research, Alfandre provides practical advice to reduce AMA discharges. "Clinicians should maintain a therapeutic alliance by approaching a patient's request to leave the hospital with openness and empathy and by eliciting the patient's preferences and competing priorities and addressing any unmet needs. They can also engage patients in the decision-making process for discharge by providing them with an expanded medically reasonable range of options instead of simply a choice to stay or leave. However, competent patients have the right to decline recommended care, so if remaining hospitalised becomes untenable, physicians should provide a sion to leave the hospital.' plantation (figure 3). medically acceptable and safe alternative treatment plan that attempts to reduce the harm associated with the patient's deci-

There is increasing use of a left ventricular assist device (LVAD) to stabilise patients with end-stage heart failure who are awaiting heart transplantation. In this issue of Heart, Parameshwar and colleagues $^{5}$ summarise the UK experience in the 342 patients who were treated with an LVAD between 2007 and 2013 in whom the median duration of support was 534 days. At 3 years follow-up, overall survival was $49.6 \%$ with a cerebrovascular accident being the most common cause of death during LVAD support. Only about $25 \%$ of patients received a heart transplantation with even fewer able to have the device explanted without heart trans-
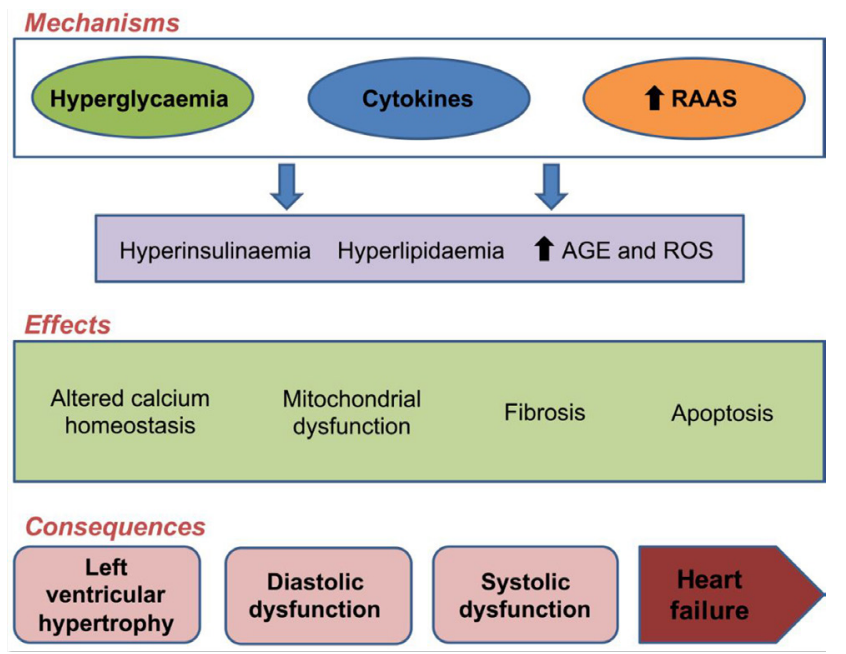

Figure 4 Potential mechanisms, effects and consequences in diabetic cardiomyopathy. AGE, advanced glycation end products; RAAS, renin-angiotensin-aldosterone system; ROS, reactive oxygen species.
In the accompanying editorial, $\mathrm{Li}$ and $\mathrm{Mahr}^{6}$ put this data in context. Heart transplantation after placement of an LVAD occurred in only $25 \%$ of these UK patients at 3 years, compared with US registry data showing transplantation rates of $50 \%$ at 2 years Although overall stroke rates appear similar in the UK and USA after LVAD implantation, stroke accounted for only $18 \%$ of deaths in the USA registry compared with $35 \%$ in the UK data. However, they go on to suggest that with the increasing use of LVADs despite no change in heart transplantation rates, the UK experience may be the future for the USA as well. They conclude: "It is our opinion that a deliberate and proactive effort to research and implement optimal medical management strategies to reduce LVAD complications, especially strokes, is urgently needed."

The Education in Heart ${ }^{7}$ article in this issue provides an excellent discussion of the definition, pathophysiology, clinical outcomes and management of patients with diabetic cardiomyopathy (figure 4).

The Image Challenge ${ }^{8}$ question shows an abdominal computed tomographic scan in a young woman with known congenital heart disease (ACHD) who presented with abdominal pain. As more patients with complex ACHD survive to adulthood, general cardiologists will need some degree of familiarly with long-term complications because these patients often will present acutely to the local medical centre.

Funding This research received no specific grant from any funding agency in the public, commercial or notfor-profit sectors.

Competing interests None declared.

Patient consent for publication Not required.

Provenance and peer review Commissioned; internally peer reviewed.

(c) Author(s) (or their employer(s)) 2019. No commercial re-use. See rights and permissions. Published by BMJ.

\section{Check for updates}

To cite Otto CM. Heart 2019;105:261-263.

Heart 2019;105:261-263.

doi:10.1136/heartjnl-2019-314705

\section{REFERENCES}

1 Nascimento BR, Beaton AZ, Nunes MCP, et al. Integration of echocardiographic screening by nonphysicians with remote reading in primary care. Heart 2019;105:283-90.

2 Peters A, Patil PV. Tele-echocardiography: enhancing quality at the point-of-care. Heart 2019;105:264-5

3 Kwok CS, Walsh MN, Volgman A, et al. Discharge against medical advice after hospitalisation for acute myocardial infarction. Heart 2019;105:315-21. 
4 Alfandre D. Advancing the science of discharges against medical advice: taking a deeper dive. Heart 2019;105:268-9.

5 Parameshwar J, Hogg R, Rushton S, et al. Patient survival and therapeutic outcome in the UK bridge to transplant left ventricular assist device population. Heart 2019:105:291-6.

6 Li S, Mahr C. Evaluating ventricular assist device outcomes internationally with a focus on neurological events. Heart 2019;105:266-7.
7 Lee MMY, McMurray JJV, Lorenzo-Almorós A, et al. Diabetic cardiomyopathy. Heart 2019;105:337-45.

8 O'Donohoe R, Fitzsimmons S, Bryant TJC. Acuteonset abdominal pain in a woman in her 30 s. Heart 2019;105:275. 